\title{
Closed-Loop Applicability of the Sign-Perturbed Sums Method
}

\author{
Balázs Csanád Csáji*
}

\author{
Erik Weyer $^{\dagger}$
}

\begin{abstract}
Sign-Perturbed Sums (SPS) is a non-asymptotic system identification method that can construct confidence regions for general linear systems. It works under mild statistical assumptions, such as symmetric and independent noise terms. The SPS confidence region includes the prediction error estimate (PEM) and, for any finite sample and user-chosen confidence probability, it contains the true system parameter with exactly the given probability. Originally, SPS was introduced for open-loop systems, this paper overviews its applicability in closed-loop setups. The three main PEM approaches of closed-loop identification are addressed: direct, indirect and joint input-output, and it is discussed whether SPS can be applied to construct guaranteed finite sample confidence regions around these PEM estimates. Some parametrization issues are also highlighted and, finally, two numerical experiments are presented demonstrating SPS for closed-loop systems.
\end{abstract}

\section{INTRODUCTION}

Standard system identification methods are typically formulated assuming open-loop systems, while in practice, for example in industry, plants usually operate in closed-loop. In many cases, because of stability, safety or efficiency reasons, experimental data can only be gathered under feedback control. Other examples include biological and economical systems where the feedback mechanism is intrinsic and hence performing open-loop experiments is not possible. Unfortunately, several identification methods perform poorly under certain experimental conditions, such as feedback control [9], [10]. Therefore investigating identification methods for closed-loop systems is of high practical importance. Moreover, having feedback control may even be advantageous in some situations [5], e.g., in "identification for control": models identified in closed-loop are more reliable in the critical zones, which are important for robust control [6].

The main problem of identifying closed-loop systems, of course, comes from the dependencies between the input signal and the noise process of the system. This may cause many standard approaches, including instrumental variable-, subspace-, correlation- and spectral analysis methods to fail in closed-loop [4]. An example of an algorithm that does work in a closed-loop setting is the prediction error method

The work of B. Cs. Csáji was supported by the Hungarian Scientific Research Fund (OTKA), pr. no. 113038, and by the János Bolyai Research Fellowship of the Hungarian Academy of Sciences, pr. no. BO/00683/12/6. The work of E. Weyer was supported by the Australian Research Council (ARC) under Discovery Grants DP0986162 and DP130104028.

* Fraunhofer Project Center at MTA SZTAKI: The Institute for Computer Science and Control, Hungarian Academy of Sciences, Kende utca 13-17, Budapest, Hungary, H-1111; balazs.csajiesztaki.mta.hu

${ }^{\dagger}$ Department of Electrical and Electronic Engineering, Melbourne School of Engineering, The University of Melbourne, 240 Grattan Street, Victoria, 3010, Australia; ewey@unimelb.edu.au
(PEM), which has three main directions to address closedloop problems: (i) the direct, (ii) the indirect and (iii) the joint input-output approach (see Section IV for more details).

In many applications, for example, those that involve strong safety, stability or quality constraints, point estimates need to be accompanied by confidence regions. These regions may serve as quality tags and are also essential for robust control design. The standard way of obtaining such regions is to build confidence ellipsoids based on the asymptotic distribution of the estimate [9], [10], which is Gaussian in the PEM case. However, this only leads to approximate results in a finite sample setting. Therefore, these confidence sets do not come with strict theoretical guarantees for finite datasets and, consequently, should be used only as heuristics.

Motivated by this, the recently developed Sign-Perturbed Sums (SPS) method [1], [2], [3], [7], [8] can build guaranteed non-asymptotic confidence regions around a given nominal estimate under mild statistical assumptions, such as independent and symmetric noise terms. Particularly, in [1] it was shown that SPS can build finite sample confidence regions for parameters of general linear systems which are centered around the PEM estimate and contain the true parameter with an exact confidence probability, which is user-chosen.

The original SPS approach assumes open-loop control, in this paper we analyze the applicability of SPS for closed-loop systems. Particularly, we address the problem of generating confidence regions around the direct, indirect and jointinput output PEM estimates of general linear systems. We analyze the construction of the appropriate SPS regions, their confidence probabilities and some parametrization issues.

\section{Problem Setting}

Let us consider the following scalar linear system [9], [10]

$$
Y_{t} \triangleq G\left(z^{-1} ; \theta^{*}\right) U_{t}+H\left(z^{-1} ; \theta^{*}\right) N_{t},
$$

where $Y_{t}$ is the output, $U_{t}$ is the input, $N_{t}$ is the noise at (discrete) time $t ; G, H$ are (causal) rational transfer functions; and $z^{-1}$ is the backward shift operator. The true parameter is $\theta^{*} \in \Theta$, where $\Theta$ is the set of allowed parameters. The system operates in closed-loop and the feedback rule is

$$
U_{t} \triangleq L\left(z^{-1} ; \eta^{*}\right) R_{t}-F\left(z^{-1} ; \eta^{*}\right) Y_{t}
$$

where $R_{t}$ could be a reference signal, setpoint or noise affecting the controller at time $t$ (or simply zero). Operators $F\left(z^{-1}, \eta^{*}\right)$ and $L\left(z^{-1}, \eta^{*}\right)$ are (causal) rational transfer functions, which are parametrized independently of (1).

Our main assumptions on the system are as follows 


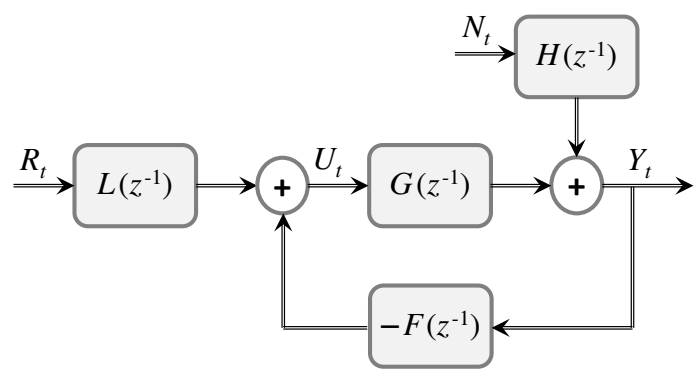

Fig. 1. General structure of the addressed systems.

A1 The "true" systems generating $\left\{Y_{t}\right\}$ and $\left\{U_{t}\right\}$ are in the model classes; $G$ and $H$ have known orders.

A2 Transfer function $H\left(z^{-1} ; \theta\right)$ has a stable inverse, and $G(0 ; \theta)=0$ and $H(0 ; \theta)=1$, for all $\theta \in \Theta$.

A3 The noise sequence $\left\{N_{t}\right\}$ is independent, and each $N_{t}$ has a symmetric probability distribution about zero.

A4 The initialization is known, $Y_{t}=N_{t}=R_{t}=0, t \leq 0$

A5 The subsystems from $\left\{N_{t}\right\}$ and $\left\{R_{t}\right\}$ to $\left\{Y_{t}\right\}$ are asymptotically stable and have no unstable hidden modes.

A6 Signal $\left\{R_{t}\right\}$ is independent of the noise $\left\{N_{t}\right\}$.

Assumption A5 implies that filters $L\left(z^{-1} ; \theta\right), H\left(z^{-1} ; \theta\right)$ and $\left(1+G\left(z^{-1} ; \theta\right) F\left(z^{-1} ; \theta\right)\right)^{-1}$ are stable [10].

Note that for most asymptotic results related to PEM we typically also assume that $\left\{R_{t}\right\}$ is persistently exciting, which is needed to ensure that the control is informative, for example, it is immediately implied if $L\left(z^{-1}, \eta^{*}\right)$ is the identity filter [9]. Sometimes the controller is allowed to be time varying, e.g., it can switch between $i=1, \ldots, r$ modes

$$
U_{t} \triangleq L_{i}\left(z^{-1} ; \eta^{*}\right) R_{t}-F_{i}\left(z^{-1} ; \eta^{*}\right) Y_{t},
$$

which helps to perform informative experiments, e.g., if there is no external signal [9], [10]. Here, for simplicity we do not consider this case, however, it is straightforward to extend the confidence region constructions to allow this.

Given a finite sample of size $n$ containing the inputs, $\left\{U_{t}\right\}$, the outputs, $\left\{Y_{t}\right\}$, and the external signal, $\left\{R_{t}\right\}$, as well as a user-chosen confidence probability $p$, we aim at building a guaranteed confidence region for the true parameter, $\theta^{*}$ (or for $\left(\theta^{*}, \eta^{*}\right)$ in case of joint input-output identification), which also contains a given nominal estimate, such as the direct, the indirect or the joint-input output PEM estimate. Before we investigate closed-loop SPS, we briefly review its construction in case of open-loop systems following [1].

\section{REVIEW OF OPEN-LOOP SPS}

In this section we assume that the system operates in openloop, namely, that $\left\{U_{t}\right\}$ and $\left\{N_{t}\right\}$ are independent.

Under our assumptions, given a particular $\theta$ the noise terms can be reconstructed from an inverted system [10],

$$
\widehat{N}_{t}(\theta) \triangleq H^{-1}\left(z^{-1} ; \theta\right)\left(Y_{t}-G\left(z^{-1} ; \theta\right) U_{t}\right),
$$

which are referred to as residuals or prediction errors. It is important to note that $\widehat{N}_{t}\left(\theta^{*}\right)=N_{t}$, for all $t$.
In this paper we concentrate on the quadratic cost criterion, thus the prediction error estimate, $\tilde{\theta}_{n}$, is defined as [9]

$$
\widehat{\theta}_{n} \triangleq \underset{\theta \in \Theta}{\arg \min } \sum_{t=1}^{n} \widehat{N}_{t}^{2}(\theta),
$$

where $\Theta$ is the class of allowed models. The prediction error estimate satisfies the following (c.f. "normal") equation

$$
\sum_{t=1}^{n} \widehat{N}_{t}\left(\widehat{\theta}_{n}\right) \psi_{t}\left(\widehat{\theta}_{n}\right)=0
$$

where $\psi_{t}(\theta)$ denotes the gradient of $\widehat{N}_{t}(\cdot)$ w.r.t. parameter $\theta$. Note that for standard polynomial models, like Box-Jenkins, there are closed-form expressions for the gradients [9], [1].

SPS builds its confidence region by perturbing equation (3): given a specific $\theta$ to test, it inverts the system by computing the prediction errors, then it builds $m-1$ alternative output trajectories using perturbed versions of the residuals,

$$
\bar{Y}_{t}\left(\theta, \alpha_{i}\right) \triangleq G\left(z^{-1} ; \theta\right) U_{t}+H\left(z^{-1} ; \theta\right)\left(\alpha_{i, t} \widehat{N}_{t}(\theta)\right),
$$

where $\left\{\alpha_{i, t}\right\}$ are $(m-1) \times n$ i.i.d. random signs, that is random variables which take values \pm 1 with probability $1 / 2$ each; and $\alpha_{i}$ denotes the vector $\left(\alpha_{i, 1}, \ldots, \alpha_{i, n}\right)$. Note that $n$ is the sample size of the residuals we can reconstruct from the observations and $m$ is a user-chosen design parameter, affecting the confidence probability, see later.

It is known that function $\psi_{t}(\theta)$ can be treated as a linear filter which takes $\left\{Y_{t}\right\}$ and $\left\{U_{t}\right\}$ as inputs, that is

$$
\psi_{t}(\theta)=W\left(z^{-1} ; \theta\right) Y_{t}+Q\left(z^{-1} ; \theta\right) U_{t},
$$

where $W$ and $Q$ are vector-valued linear filters.

Then, we produce $m-1$ perturbed versions of $\psi_{t}(\theta)$ by

$$
\bar{\psi}_{t}\left(\theta, \alpha_{i}\right) \triangleq W\left(z^{-1} ; \theta\right) \bar{Y}_{t}\left(\theta, \alpha_{i}\right)+Q\left(z^{-1} ; \theta\right) U_{t},
$$

and define a reference and $m-1$ sign perturbed functions

$$
\begin{aligned}
& S_{0}(\theta) \triangleq \Psi_{n}^{-\frac{1}{2}}(\theta) \sum_{t=1}^{n} \psi_{t}(\theta) \widehat{N}_{t}(\theta) \\
& S_{i}(\theta) \triangleq \bar{\Psi}_{n}^{-\frac{1}{2}}\left(\theta, \alpha_{i}\right) \sum_{t=1}^{n} \alpha_{i, t} \bar{\psi}_{t}\left(\theta, \alpha_{i}\right) \widehat{N}_{t}(\theta),
\end{aligned}
$$

where $i \in\{1, \ldots, m-1\}$, and matrices $\Psi_{n}$ and $\bar{\Psi}_{n}\left(\theta, \alpha_{i}\right)$ are (perturbed) covariance estimates defined by [1]

$$
\begin{aligned}
\Psi_{n}(\theta) & \triangleq \sum_{t=1}^{n} \psi_{t}(\theta) \psi_{t}^{\mathrm{T}}(\theta), \\
\bar{\Psi}_{n}\left(\theta, \alpha_{i}\right) & \triangleq \sum_{t=1}^{n} \bar{\psi}_{t}\left(\theta, \alpha_{i}\right) \bar{\psi}_{t}^{\mathrm{T}}\left(\theta, \alpha_{i}\right),
\end{aligned}
$$

which are primarily used to shape the confidence region, but they do not affect the confidence probability.

Testing whether a particular parameter $\theta$ is in the SPS confidence region is based on the rank of $\left\|S_{0}(\theta)\right\|^{2}$ in the ordering of variables $\left\{\left\|S_{i}(\theta)\right\|^{2}\right\}$. Since, there could be ties, we introduce a strict total order with the help of a uniform random permutation. More precisely [3], let $\nu$ be a 
random permutation on $\{0, \ldots m-1\}$. Given $m$ real numbers $Z_{0}, \ldots, Z_{m-1}$ we define a strict total order $\succ_{\nu}$ as follows

$$
\begin{gathered}
Z_{k} \succ_{\nu} Z_{j} \quad \text { if and only if } \\
\left(Z_{k}>Z_{j}\right) \text { or }\left(Z_{k}=Z_{j} \text { and } \nu(k)>\nu(j)\right) .
\end{gathered}
$$

We denote the rank of $\left\|S_{0}(\theta)\right\|^{2}$ in the ordering (w.r.t. $\succ_{\nu}$ ) of the variables $\left\{\left\|S_{i}(\theta)\right\|^{2}\right\}$ by $\mathcal{R}(\theta)$. For example, $\mathcal{R}(\theta)=1$ if $\left\|S_{0}(\theta)\right\|^{2}$ is the smallest in the ordering of $\left\{\left\|S_{i}(\theta)\right\|^{2}\right\}$, $\mathcal{R}(\theta)=2$ if it is the second smallest and so on.

Then, the SPS confidence region is constructed by

$$
\widehat{\Theta}_{n} \triangleq\{\theta \in \Theta: \mathcal{R}(\theta) \leq m-q\},
$$

where $m>q>0$ are user-chosen integer parameters.

One of the main advantages of SPS is that its confidence region has exact confidence probability: it can be proven [1] that under assumptions A1, A2, A3, A4 and assuming openloop inputs, the coverage probability of $\theta^{*}$ is exactly ${ }^{1}$

$$
\mathbb{P}\left(\theta^{*} \in \widehat{\Theta}_{n}\right)=1-\frac{q}{m} .
$$

Since $m$ and $q$ are user-chosen parameters, the confidence probability is under our control. Moreover, since the PEM estimate, $\widehat{\theta}_{n}$, satisfies equation (3), we have $\left\|S_{0}\left(\widehat{\theta}_{n}\right)\right\|^{2}=0$ implying that the PEM estimate is always included in the SPS confidence region, assuming it is non-empty.

In the special case of linear regression problems, such as generalized finite-impulse response (FIR) systems, it can be proved, as well, that the SPS confidence region is strongly consistent [2] and it is also star convex with the least-squares estimate as a star center [3]. Nevertheless, the main strength of SPS lies in the non-conservativeness of its confidence sets.

\section{CLOSED-Loop SPS}

In this section we turn our attention to build SPS confidence regions for closed-loop systems around various PEM estimates. There are three main approaches to estimate closed-loop systems with PEM [4], [9], [10], namely

1) Direct Identification: The goal is to estimate $\theta^{*}$, i.e., to find $H$ and $G$. The standard (open-loop) PEM is applied on the original system and the existence of feedback is neglected. We will refer to this as direct PEM.

2) Indirect Identification: The goal is again to estimate $\theta^{*}$, i.e., to identify $H$ and $G$. It is assumed that the reference signal $\left\{R_{t}\right\}$ is measurable and the control mechanisms, $F$ and $L$, are known. Under this assumptions, the system can be traced back to an open-loop system (by treating the reference signal as the input) and an open-loop PEM estimate can be computed using the modified system. We will refer to this as indirect PEM.

3) Joint Input-Output Identification: The goal is to estimate $\left(\theta^{*}, \eta^{*}\right)$, namely to identity both the system given by $H$ and $G$ as well as the controller $F$. For this case, we will assume that there is no reference signal. The system is

\footnotetext{
${ }^{1}$ The original proof used a slightly different tie-breaking rule, however, they are equivalent hence this does not affect the confidence probability.
}

analyzed as if both $\left\{Y_{t}\right\}$ and $\left\{U_{t}\right\}$ were outputs. We will refer to this approach as input-output PEM.

In what follows we investigate SPS for these PEM variants.

\section{A. Direct Identification}

Direct PEM, i.e., when we disregard the feedback and treat the system as it operated in open-loop, has several advantages [9], e.g., it works regardless of the complexity of the regulator which can be unknown, it does not require special algorithms, consistency and optimal accuracy can be obtained and even unstable systems can be handled.

Following these ideas, we can try to apply SPS to build confidence sets in the same way: by simply neglecting the controller. We will refer to this approach as naive SPS.

We have to be careful though, since standard SPS as described by Section III does not lead to guaranteed confidence regions for closed-loop setups. The reason for this is that

$$
\bar{Y}_{t}\left(\theta, \alpha_{i}\right) \triangleq G\left(z^{-1} ; \theta\right) U_{t}+H\left(z^{-1} ; \theta\right)\left(\alpha_{i, t} \widehat{N}_{t}(\theta)\right),
$$

does not produce valid alternative output trajectories in case the perturbed noises are applied. Namely, in case the past outputs are different, $\left\{U_{t}\right\}$ should be different, as well, because of the closed-loop nature of the problem. This means that $\left\{\left\|S_{i}\left(\theta^{*}\right)\right\|^{2}\right\}$ will not have the same distribution for all $i \in\{0, \ldots, m-1\}$, since the feedback is neglected for $i=1, \ldots, m-1$, while the original system, represented by $i=0$, took it into account when the outputs were generated.

Since the conditional i.i.d. nature of $\left\{\left\|S_{i}\left(\theta^{*}\right)\right\|^{2}\right\}$ variables is the key element of proving the exact confidence probability of SPS sets, this result is not guaranteed for naive SPS.

A natural approach to solve this is to produce alternative input trajectories, too. A disadvantage of doing so is that it requires access to the controller. On the other hand, we do not need an explicit model, e.g., a closed-form expression, for the regulator (it may even be nonlinear), we only need to be able to simulate it for some (alternative) outputs.

In this case the alternative trajectories are $\widetilde{Y}_{t}\left(\theta, \alpha_{i}\right) \triangleq G\left(z^{-1} ; \theta\right) \bar{U}_{t}\left(\theta, \alpha_{i}\right)+H\left(z^{-1} ; \theta\right)\left(\alpha_{i, t} \widehat{N}_{t}(\theta)\right)$, where, assuming the controller is (2), input $\bar{U}_{t}\left(\theta, \alpha_{i}\right)$ is

$$
\bar{U}_{t}\left(\theta, \alpha_{i}\right) \triangleq L\left(z^{-1} ; \eta^{*}\right) R_{t}-F\left(z^{-1} ; \eta^{*}\right) \bar{Y}_{t}\left(\theta, \alpha_{i}\right),
$$

note that this does not lead to an algebraic loop, since there is a delay in the controller (A2), i.e., $G(0 ; \theta)=0$.

Then, we redefine the alternative versions of $\psi_{t}(\theta)$ by

$$
\widetilde{\psi}_{t}\left(\theta, \alpha_{i}\right) \triangleq W\left(z^{-1} ; \theta\right) \tilde{Y}_{t}\left(\theta, \alpha_{i}\right)+Q\left(z^{-1} ; \theta\right) \bar{U}_{t}\left(\theta, \alpha_{i}\right) .
$$

The rest of the process of building the SPS region follows Section III, the only change is that we replace functions $\left\{\bar{\psi}_{t}\left(\theta, \alpha_{i}\right)\right\}$ with the modified $\left\{\widetilde{\psi}_{t}\left(\theta, \alpha_{i}\right)\right\}$. The confidence probability $1-q / m$ is again exact; and a very similar proof can be used as before (see the Appendix of [1]) with treating $\left\{Y_{t}\right\}$ as the output and the reference signal $\left\{R_{t}\right\}$ as the input. Also, since the direct PEM estimate satisfies equation (3), it is always included in the SPS confidence region, assuming it is non-empty. We call this method direct SPS. 


\section{B. Indirect Identification}

The idea of indirect PEM is to rewrite the original (1)-(2) in an alternative way as an open-loop system [10], that is

$$
Y_{t}=G_{0}\left(z^{-1} ; \kappa^{*}\right) R_{t}+H_{0}\left(z^{-1} ; \kappa^{*}\right) N_{t},
$$

where filters $G_{0}\left(z^{-1} ; \kappa^{*}\right)$ and $H_{0}\left(z^{-1} ; \kappa^{*}\right)$ are defined by

$$
\begin{aligned}
& G_{0}\left(z^{-1} ; \kappa^{*}\right) \triangleq(1+G F)^{-1} G L, \\
& H_{0}\left(z^{-1} ; \kappa^{*}\right) \triangleq(1+G F)^{-1} H,
\end{aligned}
$$

where, for simplicity, we omitted the dependence on the backward shift operator, $z^{-1}$, and the true parameters, $\theta^{*}$ and $\eta^{*}$, from the notation of the filters $G, H, F$ and $L$. In this approach it is assumed that $F$ and $L$ are known. The new filters $G_{0}\left(z^{-1} ; \kappa\right)$ and $H_{0}\left(z^{-1} ; \kappa\right)$ producing the outputs can be parametrized differently than the original filters.

Since the new system (4) is open-loop (A6), with inputs $\left\{R_{t}\right\}$ and outputs $\left\{Y_{t}\right\}$, the standard SPS construction for open-loop systems, presented in Section III, can be used to build a confidence region around the indirect PEM estimate. The difference is that we must use the alternative system (4) and identify the parameters of $G_{0}$ and $H_{0}$, namely $\kappa^{*}$.

If filters $G_{0}$ and $H_{0}$ are not parametrized by $\theta$, the new parameters $\kappa$, to be tested by SPS, can be computed from $\theta$. For example, if $\theta$ are the potential parameters, we should compute $\kappa$ parametrizing $G_{0}$ and $H_{0}$ from

$$
\begin{aligned}
(1+G(\theta) F)^{-1} G(\theta) L & =G_{0}(\kappa), \\
(1+G(\theta) F)^{-1} H(\theta) & =H_{0}(\kappa) .
\end{aligned}
$$

Assuming we have an (exact or approximate) solution given by $\kappa=g(\theta)$ the SPS confidence set construction should take this parameter transformation into account, that is

$$
\widehat{\Theta}_{n}^{i d} \triangleq\{\theta \in \Theta: \mathcal{R}(g(\theta)) \leq m-q\},
$$

where otherwise the construction is the same as in Section III, assuming the external signal, $\left\{R_{t}\right\}$, is treated as the input, instead of $\left\{U_{t}\right\}$, i.e., system (4) is identified.

In this case, we need to following assumption to guarantee the exact confidence of the indirect SPS region

\section{A7 Parameter transformation $g$ satisfies $g\left(\theta^{*}\right)=\kappa^{*}$.}

A7 is clearly satisfied if, e.g., we also use $\theta$ to parametrize $G_{0}$ and $H_{0}$, i.e., if $g$ is the identity transformation, which is the simplest approach to take, but may complicate the parametrization. Naturally, transformation $g$ will affect the shape of the confidence region and should be chosen carefully. Furthermore, based on the usual "root of the normal equation" argument, the indirect PEM estimate is always included in the indirect SPS set, assuming it is non-empty.

Some notes on parametrization are due. Henceforth in this subsection we focus on ARMAX systems. Assume that $L$ is the identity, and (the known) $F$ can be factorized as

$$
F\left(z^{-1}\right)=\frac{D\left(z^{-1}\right)}{K\left(z^{-1}\right)}
$$

where the polynomials $D$ and $K$ are coprime. Also assume that (1) has an ARMAX model structure, that is

$$
A\left(z^{-1} ; \theta\right) Y_{t}=B\left(z^{-1} ; \theta\right) U_{t}+C\left(z^{-1} ; \theta\right) N_{t},
$$

as well as the indirect system (4) takes an ARMAX form,

$$
A_{0}\left(z^{-1} ; \kappa\right) Y_{t}=B_{0}\left(z^{-1} ; \kappa\right) R_{t}+C_{0}\left(z^{-1} ; \kappa\right) N_{t},
$$

then, we may get polynomials $A, B, C$ by solving

$$
\begin{aligned}
& A_{0}\left(z^{-1} ; \kappa\right)=A\left(z^{-1} ; \theta\right) K\left(z^{-1}\right)+B\left(z^{-1} ; \theta\right) D\left(z^{-1}\right), \\
& B_{0}\left(z^{-1} ; \kappa\right)=B\left(z^{-1} ; \theta\right) K\left(z^{-1}\right), \\
& C_{0}\left(z^{-1} ; \kappa\right)=C\left(z^{-1} ; \theta\right) K\left(z^{-1}\right) .
\end{aligned}
$$

Assuming $\theta$ is known, this leads to a system of linear equations for the unknown $\kappa$ parameters, which then can be calculated or estimated [5], if we have "enough" equations. This may not be the case if the regulator is not complex enough: it is known that a closed-loop experiment may be non-informative even if the input signal itself is persistently exciting and the regulator is perfectly known (c.f. Example 13.3 of [9]). Closed-loop identifiability is harder if we do not have a reference signal. The reader may consult Complement 10.1 of [10] for necessary and sufficient conditions for the identifiability of closed-loop ARMAX systems.

Finally, we argue that it has an advantage of using the canonical parametrization for (6), especially if there is no reference signal, even if it requires solving a system of linear equations to get the parameters of (5). Namely, this ensures that asymptotically the true system parameter will be the only critical point of the (quadratic) cost function (c.f. Complement 7.6 of [10]). This is not only an advantage from the viewpoint of finding the PEM estimate numerically, but also preferable for building SPS regions as they, by construction, include all critical points of the cost function.

\section{Joint Input-Output Identification}

In case of joint input-output identification, the controller is also unknown and should be identified, i.e., both $\theta^{*}$ and $\eta^{*}$ need to be estimated. Nevertheless, the structure of the controller should be known, including the orders of its polynomials. Here, for simplicity, we assume that there is no reference signal $\left(R_{t} \equiv 0\right)$. The core idea is to regard $\left(Y_{t}, U_{t}\right)^{\mathrm{T}}$ as the output of a two dimensional time series, and identify its parameters by open-loop PEM techniques.

Let $Z_{t} \triangleq\left(Y_{t}, U_{t}\right)^{\mathrm{T}}$ and consider the following

$Z_{t}=\left[\begin{array}{c}Y_{t} \\ U_{t}\end{array}\right]=\left[\begin{array}{c}(I+G F)^{-1} H \\ -F(I+G F)^{-1} H\end{array}\right] N_{t}=\widetilde{H}\left(z^{-1}, \kappa^{*}\right) N_{t}$,

which is an autonomous system driven by an independent sequence of symmetric noise terms, $\left\{N_{t}\right\}$. In order to build a joint confidence region for $\left(\theta^{*}, \eta^{*}\right)$, a MIMO extension of SPS would be needed. This is in fact possible and also leads to confidence regions with exact guarantees, for example, it is not hard to generalize the proof in [1] for the multidimensional case. However, we will leave this extension for further research along with other open issues, such as finding a preferable parametrization for joint input-output SPS, which ensures well-shaped confidence regions. 


\section{Simulation Supported EXAMPLES}

In this section we present two simple examples where SPS is applied to closed-loop systems. First, an ARX system is investigated, where the regulator is driven by an observed reference signal. This system is estimated by direct and indirect PEM methods for which direct and indirect SPS regions are constructed. Then, we present an ARMAX system regulated without an external reference signal, for which a direct PEM estimate is calculated with its direct SPS confidence region.

\section{A. Closed-loop ARX System with Reference Signal}

In this experiment, an ARX system generated the observations using a linear controller with a reference, that is

$$
\begin{aligned}
& y_{t} \triangleq a^{*} y_{t-1}+b^{*} u_{t-1}+n_{t} \\
& u_{t} \triangleq r_{t}-c^{*} y_{t}
\end{aligned}
$$

where $\left\{n_{t}\right\}$ was and i.i.d. sequence of standard normal variables (i.e., zero mean Gaussian variables with unit variance), $a^{*}=0.44$ and $b^{*}=0.33$ were the unknown system parameters we estimated, $c^{*}=0.5$ was the parameter of the controller (assumed to be known in the indirect case) and $\left\{r_{t}\right\}$ was an observed reference signal which was generated by an autoregressive system defined as

$$
r_{t} \triangleq d^{*} r_{t-1}+w_{t}
$$

where $\left\{w_{t}\right\}$ were i.i.d. noise variables distributed uniformly on $(0,1)$, and $d^{*}=0.9$ was an unknown constant.

In case of direct PEM, we simply neglect the presence of feedback and try to estimate (7a), while in the corresponding direct SPS method we assume that we can simulate the controller and use it when we generate alternative samples.

On the other hand, the indirect PEM approach builds on the knowledge of the controller, e.g., parameter $c^{*}$ is known, and that the system (7a)-(7b) can be rewritten as

$$
\begin{aligned}
& y_{t}=\left(a^{*}-b^{*} c^{*}\right) y_{t-1}+b^{*} r_{t-1}+n_{t}, \\
& u_{t}=r_{t}-a^{*} r_{t-1}+\left(a^{*}-b^{*} c^{*}\right) u_{t-1}-c^{*} n_{t},
\end{aligned}
$$

where (8a) is an open-loop system, i.e., it can be written as

$$
y_{t}=\bar{a} y_{t-1}+\bar{b} r_{t-1}+n_{t},
$$

where $\bar{a} \triangleq\left(a^{*}-b^{*} c^{*}\right), \bar{b}=b^{*}$ and $\left\{r_{t}\right\}$ is independent of the outputs $\left\{y_{t}\right\}$. As it was highlighted, it is the preferred parametrization for indirect SPS. This, of course, means a parameter transformation from the viewpoint of the original problem, thus it should be inverted to get a confidence region for the original parameter. Since the controller is known, e.g. $c^{*}$ is available, this can be done by solving a system of linear equations. For this particular example, it meas that an exact confidence region for $\left(a^{*}, b^{*}\right)^{\mathrm{T}}$ can be built using the samples of the reference signal, $\left\{r_{t}\right\}$, and the outputs, $\left\{y_{t}\right\}$, by

$$
\widehat{\Theta}_{n}^{i d}=\left\{(a, b)^{\mathrm{T}} \in \mathbb{R}^{2}: \mathcal{R}\left(\left(a-b c^{*}, b\right)^{\mathrm{T}}\right) \leq m-q\right\},
$$

for some user-chosen integers $m>q>0$. In our experiments $m=100, q=5$ was used leading to $95 \%$ confidence.

The results of building direct and indirect 95\% SPS confidence regions for the parameters $\left(a^{*}, b^{*}\right)$ of system

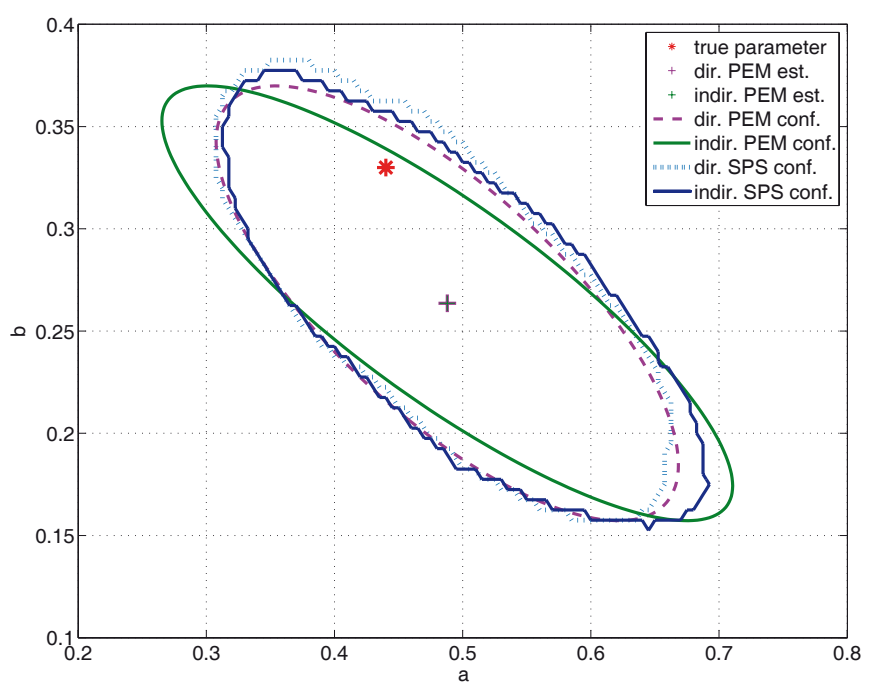

Fig. 2. $95 \%$ confidence regions $(n=100)$ with the "true" parameter " $\star$ " and the (direct, indirect) PEM estimate(s) "+". The dashed lines correspond to direct regions, while the solid lines show indirect confidence sets. In both cases, the ellipsoids show the approximate regions of the PEM estimate(s), while the curvy (light and dark blue) lines show the corresponding SPS sets.

(7a) based on $n=100$ observations are illustrated in Figure 2. The figure also presents the direct and indirect PEM estimates, which in this case coincide and are simply the least-squares estimate, with their asymptotic confidence ellipsoids. It can be observed that the shape and size of both SPS confidence regions are similar to their asymptotic counterparts. For this experiment the direct and indirect SPS provided confidence regions having similar size and shape.

\section{B. Closed-loop ARMAX System without External Signal}

In our second experiment an ARMAX system was used which was controlled without an external reference signal,

$$
\begin{aligned}
& y_{t} \triangleq a^{*} y_{t-1}+b^{*} u_{t-1}+c^{*} n_{t-1}+n_{t}, \\
& u_{t} \triangleq d^{*} y_{t}+e^{*} y_{t-1},
\end{aligned}
$$

where $\left\{n_{t}\right\}$ was an i.i.d. sequence of standard normal random variables, $a^{*}=0.33, b^{*}=0.22$, and $c^{*}=0.15$ were the unknown system parameters we wanted to estimate, and $d^{*}=0.31, e^{*}=0.23$ were the parameters of the controller, which were assumed to be known for the current experiment.

The direct PEM approach is again to simply use the samples $\left\{u_{t}\right\}$ and $\left\{y_{t}\right\}$ without taking feedback into account and estimate directly the ARMAX parameters $\left(a^{*}, b^{*}, c^{*}\right)^{\mathrm{T}}$.

In order to build the direct SPS confidence region around the direct PEM estimate, first, we need to invert the noise. In this case, the polynomials of the system are

$$
\begin{aligned}
& A\left(z^{-1}, \theta^{*}\right) \triangleq 1-a^{*} z^{-1}, \\
& B\left(z^{-1}, \theta^{*}\right) \triangleq b^{*} z^{-1} \\
& C\left(z^{-1}, \theta^{*}\right) \triangleq 1+c^{*} z^{-1}
\end{aligned}
$$

where $\theta^{*}=\left(a^{*}, b^{*}, c^{*}\right)^{\mathrm{T}}$. Then, the reconstructed noise is

$$
\hat{n}_{t}(\theta)=C^{-1}\left(z^{-1}, \theta\right)\left(A\left(z^{-1}, \theta\right) y_{t}-B\left(z^{-1}, \theta\right) u_{t}\right),
$$




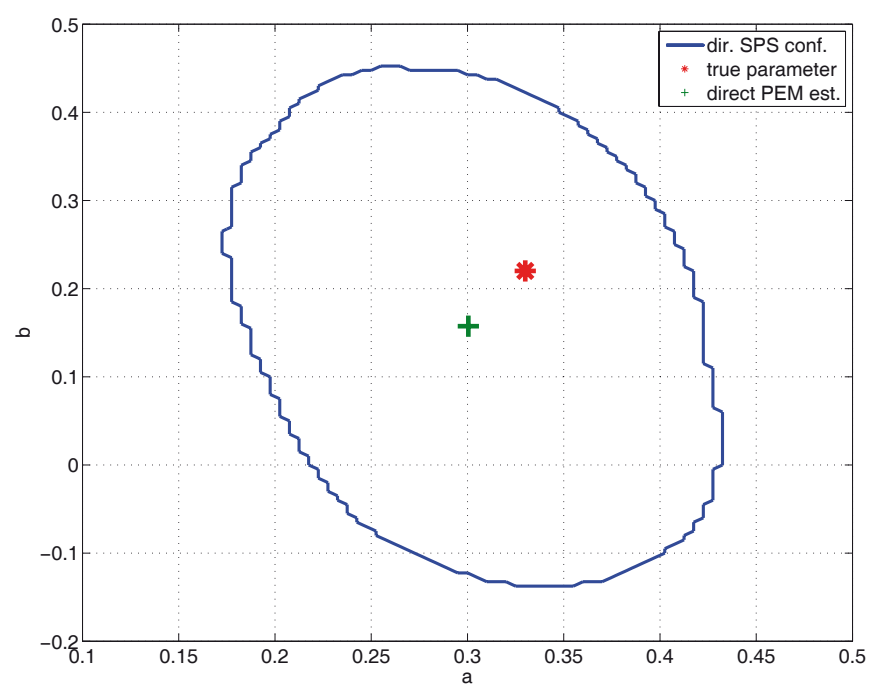

Fig. 3. Part of a $95 \%$ confidence region $(n=1000)$ for an ARMAX system with the "true" parameter " $\star$ " and the direct PEM estimate "+". The solid blue line shows the boundary of the direct SPS confidence region. The figure is drawn with parameter $c$ fixed at its direct PEM estimate.

where $\theta=(a, b, c)^{\mathrm{T}}$ is a generic parameter. This formula can be expressed for the current example as follows

$$
\hat{n}_{t}(\theta)=\sum_{k=0}^{t-2}(-1)^{k} c^{k}\left(y_{t-k}-a y_{t-k-1}-b u_{t-k-1}\right),
$$

where we also used the assumption on the initialization (A4).

The alternative output and input trajectories are

$$
\begin{aligned}
\tilde{y}_{t}\left(\theta, \alpha_{i}\right) \triangleq & a \tilde{y}_{t-1}\left(\theta, \alpha_{i}\right)+b \bar{u}_{t-1}\left(\theta, \alpha_{i}\right) \\
& +c \alpha_{i, t-1} \hat{n}_{t-1}(\theta)+\alpha_{i, t} \hat{n}_{t}(\theta), \\
\bar{u}_{t}\left(\theta, \alpha_{i}\right) \triangleq & d^{*} \tilde{y}_{t}\left(\theta, \alpha_{i}\right)+e^{*} \tilde{y}_{t-1}\left(\theta, \alpha_{i}\right),
\end{aligned}
$$

where $\left\{\alpha_{i, t}\right\}$ are i.i.d. random signs, see Section III, and $i \in\{1, \ldots, m-1\}$, hence $m-1$ alternative samples are generated. The filters $W$ and $Q$ representing the gradient of the noise as a function of $\theta$ can be expressed using the wellknown formulas (see Section 10.3 of [9]), which we omit due to lack of space. Then, we have all we need to define functions $\left\{S_{i}(\theta)\right\}$ and build the SPS confidence region.

Note that this SPS approach constructs the confidence region directly, and therefore there is no need for parameter transformations like in the previous indirect example.

Figure 3 presents a simulation using $n=1000$ observations and $m=100$ trajectories (the original one and 99 alternative, perturbed versions). We set $q=5$ ensuring exactly $95 \%$ confidence probability. Since we had three parameters to estimate, the figure only shows a projection of the set, namely we kept $c$ fixed at its direct PEM estimate.The result illustrates that the obtained non-conservative SPS confidence region is well-shaped and concentrates around the direct PEM estimate, denoted by "+" in the figure.

\section{SUMmARY AND CONCLUSION}

Extending system identification methods to closed-loop setups has high practical importance, as there are several systems which can only be observed under feedback control, especially in industry, economics and biology. Here we have investigated the closed-loop applicability of the recently developed Sign-Perturbed Sums (SPS) method [1], [2], [3], which was originally developed for open-loop systems.

We have focused on prediction error methods (PEMs) and studied how SPS can be applied to build confidence regions around the main closed-loop PEM estimates, namely, the direct-, the indirect- and the joint input-output estimate.

For open-loop systems it is known that SPS can build non-asymptotic confidence regions around the PEM estimate under very mild assumptions. The confidence regions built by SPS have exact confidence probabilities independently of the particular distribution of the noise terms, assuming they are independent and distributed symmetrically about zero.

We have argued that this favorable non-conservative property of SPS carries over to the closed-loop case. We have discussed that one can build an exact confidence region around the direct PEM estimate, in case the controller can be simulated, which is needed to generate alternative trajectories fundamental to SPS. We have explained that the exact confidence probability of indirect SPS simply follows from the previously mentioned result on open-loop systems assuming, e.g., that the indirect system uses the same parameters as the original one. The joint input-output case have been discussed, too, however as it requires a MIMO extension of SPS, it was left for further research. Finally, two simulation experiments have been presented illustrating the closed-loop applicability of SPS on simple ARX and ARMAX systems.

\section{ACKNOWLEDGMENT}

The authors would like to thank Marco C. Campi for the valuable discussions on the Sign-Perturbed Sums method.

\section{REFERENCES}

[1] B.Cs. Csáji, M.C. Campi, and E. Weyer. Sign-perturbed sums (SPS): A method for constructing exact finite-sample confidence regions for general linear systems. In 51st IEEE Conference on Decision and Control, Maui, Hawaii, pages 7321-7326, 2012.

[2] B.Cs. Csáji, M.C. Campi, and E. Weyer. Strong consistency of the sign-perturbed sums method. In 53rd IEEE Conference on Decision and Control, Los Angeles, California, pages 3352-3357, 2014.

[3] B.Cs. Csáji, M.C. Campi, and E. Weyer. Sign-perturbed sums: A new system identification approach for constructing exact non-asymptotic confidence regions in linear regression models. IEEE Transactions on Signal Processing, 63(1):169-181, 2015.

[4] U. Forssell. Closed-loop identification: Methods, theory, and applications. Linköping University Electronic Press, 1999.

[5] U. Forssell and L. Ljung. Closed-loop identification revisited. Automatica, 35(7):1215-1241, 1999.

[6] A. Karimi and I. D. Landau. Comparison of the closed-loop identification methods in terms of the bias distribution. Systems \& control letters, 34(4):159-167, 1998.

[7] M. Kieffer and E. Walter. Guaranteed characterization of exact non-asymptotic confidence regions as defined by LSCR and SPS. Automatica, 49:507-512, 2013.

[8] S. Kolumbán, I. Vajk, and J. Schoukens. Perturbed datasets methods for hypothesis testing and structure of corresponding confidence sets. Automatica, 51:326-331, 2015.

[9] L. Ljung. System Identification: Theory for the User. Prentice-Hall, Upper Saddle River, 2nd edition, 1999.

[10] T. Söderström and P. Stoica. System Identification. Prentice Hall International, Hertfordshire, UK, 1989. 